\title{
SATISFACCIÓN DE LOS ESTUDIANTES RESPECTO A LAS ACCIONES FORMATIVAS E-LEARNING EN EL ÁMBITO UNIVERSITARIO.
}

\section{STUDENTS' SATISFACTION WITH THE E-LEARNING EDUCATIONALAT THE UNIVERSITY LEVEL.}

\author{
MSc. Renata Curci La Rocca \\ rcurci@unimet.edu.ve
}

Universidad Metropolitana. Facultad de Ciencias y Artes. Departamento de Química Final Av. Boyacá con Autopista Petare Guarenas, Edificio Corimón, Urbanización Terrazas del Ávila, Caracas (Venezuela)

En los últimos años se han incrementado las investigaciones que buscan conocer el grado de satisfacción del alumnado respecto a las acciones formativas soportadas en e-learning, sobre todo en el ámbito universitario. Este artículo analiza los resultados de un estudio que perseguía recoger las percepciones que los alumnos tienen respecto al e-learning en una Universidad latinoamericana. Más concretamente, en aspectos relacionados con la asignatura de manera general, el profesor/tutor, los contenidos, los procesos de comunicación, y la plataforma utilizada en la Universidad.

Palabras clave: Satisfacción del alumnado, educación superior, e-learning, blended-learning.

In the last years researches that seek to know the degree of satisfaction of the students in relation with the educational actions supported by e-learning, especially at the University level, have been increasing. This article analyses the results of a study which collects the perceptions that the students have regarding e-learning in a Latin-American university. In particular, in aspects related to the subject, the professor/tutor, the contents, the process of communication, and the e-learning platform itself.

Keywords: Students' satisfaction, higher education, e-learning, blended-learning. 


\section{Introducción.}

En el ámbito educativo los alumnos o participantes de una acción formativa a través de la red no son simples receptores o usuarios de un servicio, como ocurre en el ámbito empresarial, son parte del proceso de enseñanza-aprendizaje. Por lo tanto, el interés de este estudio es conocer el grado de satisfacción de los estudiantes de una Universidad ubicada en Latinoamérica, la Universidad Metropolitana, durante su proceso de formación a través de Internet.

El término satisfacción del estudiante es uno de los más difíciles de delimitar en el área de la educación, tanto en la formación en modalidad presencial como en la que se ofrece a través de la red. No existe consenso ni en su definición teórica ni tampoco en la forma de medirlo.

Existen diferentes estudios que han abordado el término satisfacción del alumno en el proceso enseñanza aprendizaje que se ofrece a través de la red (Noé, 2003, citado por Llorente, 2008a; Llorente, 2008b; Llorente \& Cabero, 2008; Cabero, 2010).

DeBourg (1999, citado en Llorente, 2008a) menciona algunos de los factores que parecen influir sobre la satisfacción del alumnado en este tipo de acciones formativas: 1) Ofrecer claras expectativas sobre las tareas del curso, 2) Responder de forma rápida a los estudiantes, 3) Favorecer la participación de los estudiantes, 4) Utilizar variedad de metodologías para ayudar a que los alumnos consigan comprender mejor el material, 5) Establecer mecanismos para que los estudiantes puedan acceder al profesor, y 6) Ofrecer retroalimentación puntual y devolver a los estudiantes sus trabajos.

Mientras que Hara y Klin (2000, citado en Llorente, 2008a) mencionan aquellos factores que provocan en los estudiantes ansiedad e insatisfacción en su proceso formativo a través de la red: 1) Falta de retroalimentación por parte del profesor, 2) Falta de criterios claros sobre lo que profesor espera, y 3) Ausencia o ambigüedad en las instrucciones.

Para seguir contribuyendo en la búsqueda de los factores, o en reforzar los ya existentes, tanto los que favorecen como los que no, a la satisfacción del estudiantado en el proceso de formación a través del e-learning, se buscará conocer en este estudio el grado de satisfacción de los estudiantes de la Universidad Metropolitana durante su formación a través de la red, específicamente en la plataforma Pl@tUM.

\section{La investigación realizada.}

En este artículo se describirá uno de los estudios que forma parte de un proyecto de investigación financiado por la Universidad Metropolitana, en el que se busca analizar y comprender la problemática de la incorporación del e-learning en sus acciones formativas; tanto desde diferentes perspectivas como por diferentes estrategias y técnicas de recogida de información y con las opiniones de los diferentes actores que de forma directa pueden intervenir en ella. Dicha investigación estaba conformada por cinco estudios, uno de ellos, el que se presentará a continuación, buscaba conocer el grado de satisfacción del alumnado respecto a las acciones formativas soportadas en e-learning. Es importante señalar en este momento, que cuando hagamos referencia a las acciones formativas de e-learning en nuestro estudio, nos estaremos refiriendo a dos tipos de modalidades, tanto la híbrida o blended learning como a la modalidad totalmente virtual e-learning. El estudio fue

Píxel-Bit. Revista de Medios y Educación. No 44. Enero 2014. ISSN: 1133-8482.

E-ISSN: 2171-7966. doi: http://dx.doi.org/10.12795/pixelbit.2014.i44.15 
realizado durante el tercer período (trimestre) del año académico 2010-2011. El número total de estudiantes en la Universidad Metropolitana para el tercer periodo del año académico 2010-2011 era de 5693. Sin embargo, únicamente el $32.6 \%$ de la población, es decir 1856 alumnos, recibieron acciones formativas a través de la plataforma Pl@tUM durante el período en estudio, de los cuales $51.2 \%$ pertenecían al nivel de pregrado y $48.8 \%$ a nivel de postgrado. Durante cada período existen estudiantes inscritos en más de una asignatura, tanto en pregrado como en postgrado, y para el momento del estudio, según la información suministrada por la dependencia encargada de la implementación del e-learning en la institución (Dirección AprenRed), el número de estudiantes inscritos por asignatura en la plataforma, era de 2675, repartidos entre las 55 asignaturas y sus 123 secciones que se estaban ofreciendo para ese momento.

El instrumento utilizado para la recogida de la información fue el cuestionario, el cual ha sido muy utilizado en diversos estudios e investigaciones para fines similares (Llorente, 2008a; Llorente \& Cabero, 2008; Mirete, García \& Sánchez, 2011; Cabero, Llorente \& Puentes, 2010; Cabero, 2010). A partir de la revisión bibliográfica realizada se decidió escoger el instrumento elaborado por Llorente (2008a) denominado CUSAUF (Cuestionario de Satisfacción de Alumnos Universitarios hacia la formación online) como base para la elaboración de nuestro cuestionario, y posteriormente se modificó en algunos términos adaptándolo al contexto de nuestra investigación, y por último fue sometido a juicio de expertos.

El cuestionario definitivo estaba constituido por 55 ítems de diferente tipología: preguntas dicotómicas, elección múltiple, tipo
Likert y de respuesta abierta. Las opciones de respuesta para la escala tenían cuatro posibilidades: Totalmente en desacuerdo, En desacuerdo, De acuerdo y Totalmente de acuerdo.

Las dimensiones que se establecieron en el cuestionario para recoger la información, fueron siete: 1) Aspectos generales del alumno, 2) Aspectos generales de la asignatura, 3) Aspectos relacionados con el profesor/tutor, 4) Aspectos relacionados con los contenidos, 5) Aspectos relacionados con la comunicación, 6) Aspectos relacionados con la plataforma o entorno virtual, y 7) Apreciaciones globales. El valor obtenido para el estadístico alfa de Cronbach fue de .957 , lo que nos indica que posee un alto grado de fiabilidad.

Con la primera dimensión se pretendía conocer, además del nivel académico que estudiaba, el nombre de la asignatura a valorar, si habían cursado alguna otra asignatura a través de Internet, cómo eran sus expectativas antes de comenzar la experiencia, la valoración que hacían entre la dinámica de trabajo y la valoración que tenía el estudiante de este tipo de formación antes de iniciar la experiencia, si se habían cumplido sus expectativas al finalizar la experiencia. $\mathrm{La}$ última dimensión Apreciaciones globales se refería a los elementos que los alumnos consideraban más adecuados e inadecuados durante el desarrollo de la acción formativa.

A los estudiantes de las asignaturas en modalidad blended-learning se les entregó el cuestionario de manera presencial, mientras que, a los estudiantes de las asignaturas en modalidad e-learning se les entregó vía correo electrónico. Una vez recogidos los instrumentos, se procedió a analizar la información obtenida a través del programa estadístico de análisis de datos cuantitativo

Píxel-Bit. Revista de Medios y Educación. No 44. Enero 2014. ISSN: 1133-8482. E-ISSN: 2171-7966. doi: http://dx.doi.org/10.12795/pixelbit.2014.i44.15 
SPSS 18.0. Los análisis que se realizaron fueron: a) Análisis estadísticos descriptivos (frecuencias, porcentajes, medias y desviaciones típicas) y b) Los estadísticos utilizados fueron Kruskal-Wallis y U de MannWhitney (Hurtado de Barrera, 2010) para el contraste de diferentes hipótesis. Los análisis cualitativos, de las respuestas asociadas a las preguntas abiertas se realizaron con el programa Atlas.ti 6.0 (Muñoz Justicia, 2005).

\section{Resultados.}

En primer lugar, debemos señalar que el número total de estudiantes que rellenaron el cuestionario fue de 837. Para comenzar el análisis de los resultados obtenidos, mencionaremos los alcanzados por los primeros 7 ítems. La primera de las preguntas del cuestionario estaba dirigida a conocer el nivel académico que el alumno estaba estudiando, y nos encontramos con la siguiente distribución: $51.4 \%$ pertenecían a nivel de pregrado y $48.6 \%$ a nivel de postgrado.

Como segunda pregunta se les pedía el nombre de la asignatura sobre la que estarían haciendo su valoración y a partir de las respuestas recibidas, se determinó que fueron recogidas respuestas sobre el total de las 55 asignaturas que se estaban ofertando para el momento del estudio, las cuales estaban distribuidas de la siguiente manera: $49.1 \%$ pertenecían al nivel de pregrado y $50.9 \%$ al nivel de postgrado. La proporción de respuesta en ambas preguntas fue muy similar en cuanto a nivel académico se refiere. Otra de las pregunta se refería a la modalidad en la que estaban cursando dicha asignatura, y se encontró que el $81.8 \%$ se ofrecían en modalidad blended-learning y el $18.2 \%$ en modalidad e-learning.
También interesaba conocer, si los alumnos que participaban en esta experiencia habían cursado o no anteriormente otras asignaturas a través de Internet, y por las respuestas obtenidas podemos decir que el $63 \%$ sí había tenido experiencias de este tipo de formación con anterioridad, y un $37 \%$ de los alumnos estaban participando en ellas por primera vez.

Las expectativas con que los alumnos iniciaron su participación en las distintas asignaturas fueron muy altas; en concreto, el $79.4 \%$ apuntó que fueron muy altas o altas. Únicamente el $20.6 \%$ indicaron que iniciaron con expectativas bajas o muy bajas. Con lo que respecta a sí se cumplieron las expectativas iniciales al finalizar la experiencia formativa en sus respectivas modalidades, los resultados alcanzados nos permiten señalar que la valoración había sido muy exitosa; ya que, el 76.4\% respondió que se habían cumplido sus expectativas, únicamente el $23.6 \%$ no estuvo de acuerdo.

Para conocer las razones o motivos por los cuales los alumnos se mostraban en desacuerdo, se les pidió que indicaran cuáles habían sido los motivos que los llevaron a responder negativamente. Las respuestas que ofrecieron las podemos clasificar en cuatro grandes categorías: asignatura, profesor/tutor, comunicación, y plataforma.

- Relacionadas con la asignatura.

Una de las quejas que fue mencionada con una mayor frecuencia por parte de los alumnos, se refería a la cantidad de los contenidos, materiales y recursos para desarrollar en la asignatura, donde en algunos casos consideraban que eran demasiados mientras que en otros mencionaron la falta de los mismos: « $<$ A lo largo del trimestre la materia suministrada a través de Pl@tUM fue escasa y de poca ayuda $>\mathrm{y}<$ Las clases se convierten en grandes cantidades de texto para lee >>.

Píxel-Bit. Revista de Medios y Educación. No 44. Enero 2014. ISSN: 1133-8482.

E-ISSN: 2171-7966. doi: http://dx.doi.org/10.12795/pixelbit.2014.i44.15 
También hicieron referencia a la baja calidad de algunos contenidos y materiales: $<\mathrm{El}$ material de apoyo suministrado no cumple con los estándares necesarios y no permiten adquirir las competencias esperadas»>. En cuanto a las actividades a realizar, sus quejas iban dirigidas en algunos casos a que debían ser más prácticas y menos teóricas « En mi caso particular considero que se debería concentrar la materia en más actividades prácticas que teóricas >, mientras que en otros casos consideraban que no habían sido suficientes «Pienso que se deben reforzar las actividades realizadas para que el alumno tenga interés en la materia s. Y la otra queja se asociaba con la desorganización y estructura de la asignatura <Incongruencias entre los objetivos y las asignaciones y las clases > .

- Relacionadas con el profesor/tutor.

En relación a las quejas en relación al profesor/tutor las más frecuentes estaban relacionadas con la poca aclaratoria de las dudas y de los planteamientos hechos, y que no hacían seguimiento a la actuación de los alumnos: «Muchas veces los profesores no hacían caso a las dudas enviadas y no respetaban los tiempos» . Y la otra queja hacía referencia al poco uso que hacen los profesores de la plataforma o de algunos componentes de la misma: $\ll$ Mis expectativas no fueron cumplidas debido a la manera en la que la profesora utilizo esta herramienta».

- Relacionadas con la comunicación.

La comunicación entre profesor/tutor y alumno, y entre los propios alumnos, fue otro de los motivos por los cuales algunos estudiantes respondieron por los que no se habían cumplido sus expectativas: «Esperaba más interacción con el profesor a través de la plataforma $\gg \mathrm{y}$ < Se debería ser más exigente de manera que los estudiantes realicen sus aportes con mayor frecuencia y de manera oportuna en los foros $>$.

- Relacionadas con la plataforma.

En relación a la plataforma las quejas estaban dirigidas a la conexión y al acceso a la plataforma, la otra se refería al tiempo de dedicación que se debe emplear en la plataforma para preparar y trabajar en la asignatura: «El acceso a Pl@ @UM es difícil algunas veces $\gg \mathrm{y}<$ Se debe utilizar mucho más tiempo de trabajo en la asignatura que en la modalidad presencial $\gg$.

Una vez mencionados los motivos apuntados por los alumnos con la asignatura, la plataforma, la comunicación, el profesor/ tutor y la percepción del tipo de enseñanza, pasaremos a ofrecer a continuación los resultados alcanzados en los siguientes 48 ítems tras la aplicación del cuestionario (46 ítems medidos con escala tipo Likert, y 2 ítems medidos con preguntas abiertas).

Lo primero, es señalar que las puntuaciones medias alcanzadas y sus respectivas desviaciones típicas se encuentran representadas en la Tabla 1. Es importante tener en cuenta, para una correcta interpretación de los resultados, que las opciones ofrecidas a los estudiantes para que realizaran sus valoraciones fueron de 1 (totalmente en desacuerdo) a 4 (totalmente de acuerdo).

La media alcanzada en todos los ítems fue de 3.13, con una desviación típica de .933, esto nos permite señalar la alta valoración por parte de los estudiantes.

Lo primero que tenemos que señalar es que en la mayoría de los 46 ítems que me muestran en la Tabla 1, superaban la puntuación media de los tres puntos indicando el acuerdo con el desarrollo de la experiencia, con la excepción de siete de ellos donde se mostraba desacuerdo. Además, las puntuaciones 


\begin{tabular}{|c|c|c|}
\hline & Media & $\begin{array}{l}\text { Desv. } \\
\text { Típ. }\end{array}$ \\
\hline Dimensión: Aspectos genera les de la asignatura & 3.35 & .836 \\
\hline El programa de la asignatura ha sido adecuado & 3.32 & .812 \\
\hline $\begin{array}{l}\text { Considero que están claramente indicadas desde el in icio las fechas de realización de las } \\
\text { actividades propuestas en la asignatura (cronograma) }\end{array}$ & 3.39 & .831 \\
\hline Considero que la asignatura tiene estructura, organización, y una planificación clara & 3.34 & .863 \\
\hline Dimensión: Aspectos genera les relacionados con el profesor-tutor & 3.09 & .984 \\
\hline El profesor me facilitó la comprensión de las cuestiones técnicas de la plataforma & 3.01 & 1.008 \\
\hline $\begin{array}{l}\text { Considero adecuada la explicación de las normas de funcionamiento por parte del profesor } \\
\text { sobre el entorno formativo }\end{array}$ & 3.10 & .934 \\
\hline El profesor de la asignatura poseía un buen dominio de la materia & 3.45 & .833 \\
\hline El profesor nos estimuló para la participación & 3.19 & .954 \\
\hline Cuando fue necesario, el profesor dio información y explicó los contenidos presentados & 3.24 & .922 \\
\hline El profesor mostró valoraciones adecuadas sobre las actividades realizadas & 3.24 & .895 \\
\hline Considero adecuada la utilización de los diferentes recursos online por parte del profesor & 3.12 & .917 \\
\hline $\begin{array}{l}\text { El profesor promueve el uso de todos los servicios que ofrece la plataforma: cartelera, } \\
\text { calendario, chat, correo, entrega de actividades, foro, publicaciones } \\
\text { El profesor de la asignatura posee un buen dominio de la plataforma }\end{array}$ & $\begin{array}{l}2.92 \\
3.20\end{array}$ & $\begin{array}{l}1.020 \\
.888\end{array}$ \\
\hline $\begin{array}{l}\text { El profesor de la a signatura posee buena disposición e interés hacia este tipo de formación } \\
\text { online }\end{array}$ & 3.13 & .940 \\
\hline El profesor de la asignatura respondió dudas y consultas de manera adecuada y oportuna & 3.18 & .958 \\
\hline $\begin{array}{l}\text { El profesor señaló la necesidad de completar el espacio de "perfil de usuario" para facilitar el } \\
\text { intercambio entre los diferentes alumnos que formábamos parte de la asignatura }\end{array}$ & 2.40 & 1.110 \\
\hline $\begin{array}{l}\text { El profesor propició la realización de las actividades para facilitar el conocimiento entre los } \\
\text { diferentes alumnos que formábamos parte de los módulos online }\end{array}$ & 2.96 & .999 \\
\hline Dimensión: Aspectos genera les relacionados con los contenidos & 3.19 & .877 \\
\hline $\begin{array}{l}\text { Los trabajos y las prácticas de las diferentes unidades y sus temas asociados han sido valiosos } \\
\text { para poner en marcha los conocimientos adquiridos }\end{array}$ & 3.16 & .865 \\
\hline Considero que el número de actividades o tareas durante el transcurso de la asignatura fueron & 3.18 & .896 \\
\hline
\end{tabular}

Tabla 1. Valoraciones medias y desviaciones típicas alcanzadas. 


\begin{tabular}{|c|c|c|}
\hline & Media & $\begin{array}{l}\text { Desv. } \\
\text { Típ. }\end{array}$ \\
\hline Considero que los casos prácticos presentados fueron adecuados & 3.20 & .901 \\
\hline Considero que las evaluaciones versaban sobre los contenidos y actividades realizadas & 3.30 & .834 \\
\hline La relación entre los objetivos y los contenidos ofrecidos fue apropiada & 3.34 & .832 \\
\hline La relación entre la temporalidad y los contenidos ofrecidos fue apropiada & 3.21 & .874 \\
\hline Los diferentes contenidos que sepresentan son actuales & 3.35 & .790 \\
\hline $\begin{array}{l}\text { El volumen de información es suficiente para la formación en los diferentes contenidos } \\
\text { presentados }\end{array}$ & 3.17 & .903 \\
\hline Los contenidos presentados han sido fáciles de comprender & 3.06 & .923 \\
\hline La originalidad de los contenidos ofrecidos creo que era adecuada & 3.13 & .857 \\
\hline Considero que el interés de los contenidos desde un punto de vista teórico era apropiado & 3.16 & .838 \\
\hline El interés de los contenidos desde un punto de vista práctico era adecuado & 3.15 & .873 \\
\hline Considero que los contenidos son agradables & 3.00 & .908 \\
\hline $\begin{array}{l}\text { Considero adecuada la calidad tanto científica como didáctica- educativa de los contenidos } \\
\text { abordados }\end{array}$ & 3.17 & .867 \\
\hline Considero que los contenidos fueron suficientes & 3.24 & .866 \\
\hline $\begin{array}{l}\text { El tiempo de dedicación que requiero para la preparación y realización de las actividades o } \\
\text { trabajos de la asignatura en la plataforma me parece adecuado }\end{array}$ & 3.07 & .934 \\
\hline Dimensión: Aspectos genera les relacionados con la comunicación & 3.00 & 1.006 \\
\hline La comunicación con el profesor ha sido fluida y oportuna & 3.16 & .952 \\
\hline $\begin{array}{l}\text { La comunicación con el profesor me ha resultado fácil mediante las herramientas de } \\
\text { comunicación: correo, foro, chat,...) }\end{array}$ & 2.94 & 1.046 \\
\hline Me ha resultado sencilla la comunicación online con el resto de mis compañeros del entorno & 2.83 & 1.047 \\
\hline $\begin{array}{l}\text { Considero que utilizo todos los servicios que ofrece la plataforma (cartelera, calendario, chat, } \\
\text { correo, entrega de actividades, foro, publicaciones) }\end{array}$ & 2.84 & 1.016 \\
\hline Considero que uso el foro de la plataforma de manera adecuada & 3.03 & 1.027 \\
\hline $\begin{array}{l}\text { Considero que uso el espacio para la entrega de "Actividades" de la plataforma de manera } \\
\text { adecuada }\end{array}$ & 3.16 & .907 \\
\hline Considero que uso el espacio "publicaciones" de la plataforma de manera adecuada. & 3.04 & .984 \\
\hline
\end{tabular}

Tabla 1. Valoraciones medias y desviaciones típicas alcanzadas. 


\begin{tabular}{|l|l|l|}
\hline & Media & $\begin{array}{l}\text { Desv. } \\
\text { Típ. }\end{array}$ \\
\hline Dimensión: Aspectos genera les relacionados con la plataforma o entorno virtual & $\mathbf{3 . 1 3}$ & $\mathbf{. 9 8}$ \\
\hline El funcionamiento técnico del entorno es fácil de comprender & 3.14 & .888 \\
\hline Considero adecuada la plataforma porque me ha resultado sencilla la navegación por ella & 3.08 & .906 \\
\hline La calidad estética del entorno (tamaño y tipo de letras, colores,...) considero es adecuada & 3.19 & .852 \\
\hline $\begin{array}{l}\text { Existe adecuación entre los diferentes elementos estéticos de la plataforma (textos, imágenes, } \\
\text { gráficos,...) }\end{array}$ & 3.17 & .855 \\
\hline Los tiempos de respuesta de la plataforma (espera para acceder a un vínculo, acceso a & 2.92 & .960 \\
\hline diferentes herramientas, etc.) han sido adecuados & & \\
\hline La descarga o subida de archivos a la plataforma ha sido sencilla & 3.12 & .891 \\
\hline Laflexibilidad de trabajar en la asignatura a cualquier hora me permitió un mejor desempeño & 3.25 & .899 \\
\hline
\end{tabular}

Tabla 1. Valoraciones medias y desviaciones típicas alcanzadas.

obtenidas en las desviaciones típicas no son muy altas, lo cual indica la semejanza en las opiniones de los estudiantes.

Del análisis de los resultados alcanzados podemos destacar que los aspectos mejor puntuados por los alumnos, fueron los relacionados con la asignatura, lo cual nos indica que la mayoría de ellas tienen un cronograma claro desde sus inicios, poseen organización, estructura y planificación, además de mostrar siempre el programa a desarrollar: «Considero que están claramente indicadas desde el inicio las fechas de realización de las actividades propuestas en la asignatura (cronograma)> (3.39), «Considero que la asignatura tiene estructura, organización, y una planificación clara $\gg(3.34) \mathrm{y} \ll$ El programa de la asignatura ha sido adecuado $\gg$ (3.32).

Los alumnos también puntuaron de forma positiva los ítems asociados con el comportamiento del profesor en cuanto a su dominio sobre la asignatura y la plataforma, al valorar de manera adecuada las actividades realizadas, al dar información y explicación sobre los contenidos y responder dudas y consultas de manera oportuna, y al estimular la participación entre ellos: « El profesor de la asignatura poseía un buen dominio de la materia» (3.45), «El profesor mostró valoraciones adecuadas sobre las actividades realizadas $\gg$ (3.24), «Cuando fue necesario, el profesor dio información y explicó los contenidos presentados» $>(3.24),<$ El profesor de la asignatura posee un buen dominio de la plataforma $>(3.20),<$ El profesor nos estimuló para la participación $\gg(3.19)$ y «El profesor de la asignatura respondió dudas y consultas de manera adecuada y oportuna > (3.18).

Otro aspecto que nos gustaría destacar, es la calidad de los contenidos transmitidos por el profesor/tutor, que como puede observarse

Píxel-Bit. Revista de Medios y Educación. No 44. Enero 2014. ISSN: 1133-8482. E-ISSN: 2171-7966. doi: http://dx.doi.org/10.12795/pixelbit.2014.i44.15 
a continuación también alcanzaron puntuaciones elevadas: <<Los diferentes contenidos que se presentan son actuales $>$ (3.35), « La relación entre los objetivos y los contenidos ofrecidos fue apropiada $>$ (3.34), «Considero que las evaluaciones versaban sobre los contenidos y actividades realizadas $\gg$ ( 3.30$),<$ Considero que los contenidos fueron suficientes $\gg(3.24),<<\mathrm{La}$ relación entre la temporalidad y los contenidos ofrecidos fue apropiada $>(3,21)$, «Considero que los casos prácticos presentados fueron adecuados $>$ (3.20) y «Considero que el número de actividades o tareas durante el transcurso de la asignatura fueron adecuadas >> (3.18).

En cuanto a la plataforma, los alumnos valoraron positivamente la flexibilidad que proporciona el uso de la misma permitiendo un mejor desempeño durante el desarrollo de la acción formativa, y además la calidad estética del entorno, como se muestra a continuación: <<La flexibilidad de trabajar en la asignatura a cualquier hora me permitió un mejor desempeño» (3.26), «La calidad estética del entorno (tamaño y tipo de letras, colores,...) considero es adecuada $>(3.19)$ y « Existe adecuación entre los diferentes elementos estéticos de la plataforma (textos, imágenes, gráficos,...) )> (3.17). Los alumnos también evaluaron de manera positiva la comunicación entre ellos y el profesor, sin embargo entre sus compañeros su valoración estuvo por debajo de la puntuación media alcanzada: «<La comunicación con el profesor ha sido fluida y oportuna > (3.16).

La última dimensión del cuestionario estaba integrada por dos preguntas abiertas, con las cuales pretendíamos recoger información para conocer qué elementos consideraban los estudiantes como más adecuados y cuáles más inadecuados para su acción formativa a través de la red. Al igual que hemos hecho con la pregunta abierta realizada con anterioridad que hacía referencia a los aspectos con los que estaban en desacuerdo, en estas dos preguntas se clasificaron las respuestas con base a una serie de categorías.

Comenzaremos mencionando la pregunta que hacía referencia a los elementos que los alumnos consideraban como los más adecuados para su formación, y las respuestas fueron clasificadas en cuatro categorías: las relacionadas con la asignatura, con la atención del profesor/tutor, con la plataforma y con la comunicación.

- Relacionadas con la asignatura.

El hecho de que la asignatura se ofrece a través de Internet, a los alumnos les parece ventajoso porque les permite trabajar en ella a cualquier hora y desde cualquier lugar, es decir, les permite una mayor flexibilidad temporal-espacial para trabajar en ella con relación a la modalidad presencial: « Poder estudiar desde tu casa y aprovechar mejor el tiempo $\gg$ y $<$ Poder estudiar a cualquier hora >. También valoraron positivamente el hecho de poder acceder a una mayor cantidad de información, documentos, contenidos, etc., ya que los encuentran alojados en la plataforma: <<Fácil acceso al contenido de la materia». También hacen alusión a la buena calidad de los contenidos: « Visualización rápida de los contenidos, material de buena calidad, gran cantidad de contenidos >>. Les parecen adecuados los tipos de actividades $\mathrm{y}$ evaluaciones que se realizan en dichas asignaturas: <LLas actividades virtuales me parecen adecuadas, y también las evaluaciones me han sido útiles para una mejor formación > .

- Relacionadas con la comunicación. 
La posibilidad de comunicación entre los integrantes de la asignatura también fue uno de los elementos que valoraron como adecuados para su formación a través de Internet. La herramienta de comunicación que mejor valoraron fue el foro, ya que esta les permite aprenden de diversos temas y además de los comentarios y aportes realizados por sus compañeros: «Creo que los foros son elementos importantes ya que aprendes sobre el tema a discutir y de los diferentes puntos de vista de tus compañeros $>$, < <Los foros fueron enriquecedores ya que en la comunicación con mis compañeros me ayudaron a afianzar las ideas o conceptos expuestos en clases >>. También consideran que existe una mayor y mejor comunicación con el profesor/tutor y entre compañeros de clase: « Mejor comunicación profesor-alumno mediante plataforma $\gg,,<$ Facilidad para discutir varios temas entre los compañeros $\gg \mathrm{y} \ll<\mathrm{Me}$ nutro mucho con las intervenciones de mis compañeros, la interacción es excelente >>.

- Relacionadas con la plataforma.

En relación a la plataforma los alumnos valoran como elementos adecuados para su formación la facilidad en el uso de la plataforma y de las herramientas que la componen: « El uso de la plataforma es eficiente y también la de los elementos que la componen: foros, publicaciones y calendario >. El hecho de que es fácil acceder y navegar a través de ella, y que tenga una buena conexión: <<La plataforma siempre está disponible $\gg \mathrm{y}<<$ El fácil acceso a la plataforma desde cualquier lugar te permite trabajar en los temas y tener acceso a toda la información y a los soportes >>. También se refieren positivamente al hecho de que se les capacite al inicio de la asignatura en el manejo de la plataforma: <<Buena presentación sobre el uso de la plataforma al inicio del curso » .
- Relacionadas con el profesor/tutor.

Los estudiantes valoran de manera positiva que los profesores hagan las aclaratorias sobre las dudas que surgen y sobre el tipo de información que se requiere, y además, que estén disponibles para ofrecerlas: < La constancia del profesor aclarando dudas $>$ y $<<$ La respuesta oportuna de las inquietudes reportadas en el foro por parte del profesor >. Como también, la dedicación con la que ofrecen este tipo de acciones formativas: «La dedicación y estimulación que genera la profesora para el uso de la plataforma〉>.

A continuación mostraremos los diferentes resultados vinculados con la siguiente pregunta, la que hacía referencia a los elementos que los alumnos consideraban como los más inadecuados para su formación. Las respuestas fueron clasificadas en cuatro categorías: las relacionadas con la asignatura, con la atención del profesor/tutor, con la comunicación y con la plataforma.

- Relaciones con la asignatura.

Esta categoría es una de las que obtuvo mayor número de críticas por parte de los alumnos, haciendo referencia a la gran cantidad de actividades y poco tiempo para ser resueltas, en algunos casos con contenido no actualizado, poco pertinente o incompleto: $<$ El volumen de actividades y trabajos es muy alta $>,<$ Baja flexibilidad en los tiempos de entrega de las asignaciones $\gg y$ y $\ll$ La baja calidad o claridad de los contenidos de la materia y sus apuntes >>.

- Relaciones con la comunicación.

Otro de los elementos inadecuados para los alumnos fue la poca comunicación con el profesor y estar conectados de manera síncrona para que ocurra y por sus horarios de trabajo se les hace difícil: « Hay muy poca comunicación sobre todo con los

Píxel-Bit. Revista de Medios y Educación. No 44. Enero 2014. ISSN: 1133-8482.

E-ISSN: 2171-7966. doi: http://dx.doi.org/10.12795/pixelbit.2014.i44.15 
compañeros $\gg, ~ «$ La poca comunicación con el profesor $\gg \mathrm{y}<$ El uso del chat puesto que por la naturaleza del postgrado que es semipresencial y las actividades propias de cada participante se hace difícil la conexión a una misma hora».

- Relacionadas con la plataforma.

Según los alumnos los elementos más inadecuados asociados con la plataforma son los problemas a nivel técnico, de lentitud o velocidad para navegar por la plataforma, y además la lentitud de conexión a Internet en el país: «Disponibilidad reducida de conexión, tiempos de respuesta lentos $\gg$. También se refirieron a la necesidad de actualización de la plataforma: <Actualizar portal para hacerlo más amigable $>y$ al exceso de tiempo que se requiere para la preparación y trabajar en la asignatura: «El tener que meterme todos los días en la plataforma, antes solo tenía que ir a clases algunos días durante la semana >.

- Relacionadas con el profesor/tutor.

En esta categoría, los estudiantes consideraron inadecuada la actuación del profesor por la falta de dedicación e interés durante el dictado de la asignatura, por no ofrecer una adecuada consulta de dudas y por no ofrecer una retroalimentación oportuna, y por último por no utilizar todas las funcionalidades de la plataforma: «No estoy en desacuerdo con la plataforma, pero sí con la forma de dar la materia, ya que no hay apoyo por parte del profesor », «Los profesores casi no usan la plataforma y tampoco aprovechan todos los usos que ella ofrece $>,<$ El hecho que el profesor no conteste las dudas $\gg$ y $<$ Lentitud en la retroalimentación por parte del profesor $\gg$.

Una vez realizados estos análisis, la siguiente parte del estudio estuvo dirigida a contrastar diferentes hipótesis estadísticas que nos planteamos, las cuales se mencionan a continuación:

- Si las valoraciones de los alumnos variaban en función de las expectativas iniciales que tenían al comenzar la acción formativa a través de la red.

- Si las valoraciones de los alumnos variaban en función de cómo ellos consideraban que había sido la relación en la dinámica de trabajo llevada a cabo en los módulos de la asignatura, y las expectativas iniciales que tenían antes de comenzar la acción formativa a través de la red.

- Si las valoraciones de los alumnos variaban en función de cómo consideraban que se habían cumplido sus expectativas al finalizar la acción formativa a través de la red.

Es importante señalar que, que en los tres casos las hipótesis estadísticas que contrastamos fueron las siguientes:

- H0 (hipótesis nula): No existen diferencias significativas entre la variable contrastada y la percepción de significación por parte del alumno, con un riesgo alfa de equivocarnos del.05.

- H1 (hipótesis alternativa): Si existen diferencias significativas entre la variable contrastada y la percepción de significación por parte del alumno, con un riesgo alfa de equivocarnos del .05 .

A continuación se presentarán los resultados más relevantes alcanzados para cada hipótesis planteada, sin presentar los diferentes estadísticos obtenidos en cada una de ellas, de manera de facilitar la lectura de los mismos.

Si las valoraciones de los alumnos variaban en función de las expectativas iniciales que tenían al comenzar la acción formativa a través de la red.

En este caso, el test estadístico que utilizamos fue la prueba de Kruskal-Wallis para 
realizar el contraste de la $\mathrm{H} 0$ y la $\mathrm{H} 1$. Los valores alcanzados nos permitieron rechazar la H0, y aceptar la hipótesis alterna (H1), con un riesgo alfa de equivocarnos inferior al .05 o inferior, para todos los ítems planteados, excepto para los que se muestran a continuación:

- Considero que están claramente indicadas desde el inicio las fechas de realización de las actividades propuestas en la asignatura (cronograma).

- El profesor de la asignatura respondió dudas y consultas de manera adecuada y oportuna.

- El profesor señaló la necesidad de completar el espacio del perfil del usuario para facilitar el intercambio entre los diferentes alumnos que formábamos parte de la asignatura.

- Los contenidos presentados han sido fáciles de comprender.

- Considero que los contenidos fueron suficientes.

- El funcionamiento técnico del entorno es fácil de comprender.

- Los tiempos de respuesta de la plataforma (espera para acceder a un vínculo, acceso a diferentes herramientas, etc.) han sido adecuados.

- La descarga o subida de archivos a la plataforma ha sido sencilla.

De manera que, para todos los ítems con la excepción de los que acabamos de mencionar, podemos inferir que las expectativas iniciales que los alumnos tenían al comenzar las acciones formativas a través de la red sí han influido en las diferentes valoraciones realizadas por ellos.

Con objeto de conocer las diferencias entre cuales grupos se daban las diferencias; se analizaron las puntuaciones de los rangos promedio para cada uno de los ítems formulados, y por los resultados

obtenidos, los alumnos que se mostraron antes de comenzar la experiencia con expectativas muy bajas o bajas son los que obtienen puntuaciones mayores, y por tanto son los que tendieron a valorar los diferentes ítems de nuestro cuestionario como estando totalmente de acuerdo o de acuerdo con ellos, después de participar en una acción formativa a través de la red.

Si las valoraciones de los alumnos variaban en función de cómo ellos consideraban que había sido la relación en la dinámica de trabajo llevada a cabo en los módulos de la asignatura, y las expectativas iniciales que tenían antes de comenzar la acción formativa a través de la red.

También en este caso, el test estadístico utilizado fue la prueba de Kruskal-Wallis. Los valores alcanzados nos permitieron rechazar la $\mathrm{H} 0$, y aceptar la hipótesis $\mathrm{H} 1$, con un riesgo alfa de equivocarnos inferior al $.05 \mathrm{o}$ inferior, para todos los ítems planteados. En consecuencia, podemos señalar que la relación entre la dinámica de trabajo llevada a cabo en los módulos de la asignatura, y las expectativas iniciales que tenían antes de comenzarlos, habían influido en las valoraciones que realizaron respecto a su participación en la acción formativa a través de la red. De los rangos promedios alcanzados, podemos inferir que los alumnos que se mostraron con expectativas muy bajas son los que obtuvieron las mayores puntuaciones, y por lo tanto son los que tendieron a valorar los diferentes ítems de nuestro cuestionario como estando totalmente de acuerdo o de acuerdo con ellos. 
Si las valoraciones de los alumnos variaban en función de cómo consideraban que se habian cumplido sus expectativas al finalizar la acción formativa a través de la red.

A diferencia de los casos anteriores, el estadístico que utilizamos fue la U de MannWhitney para realizar el contraste de la $\mathrm{H} 0 \mathrm{y}$ la H1. En todos los casos rechazamos la H0 formuladas y aceptamos la $\mathrm{H} 1$, con un riesgo alfa de equivocarnos inferior al .05. Por lo que podemos decir que hay relación entre las valoraciones que realizaron los estudiantes de la serie de ítems planteados, y sus percepciones de si se cumplieron o no, sus expectativas sobre la formación a través de la red. Los valores alcanzados nos permitieron señalar que aquellos estudiantes que respondieron que no se cumplieron las expectativas que tenían al inicio de su participación en la experiencia, son los que puntuaron de forma más baja, y por tanto, más cercanas al totalmente en desacuerdo o en desacuerdo los diferentes ítems que se les ofrecieron. Es decir, los estudiantes que no vieron cumplidas sus expectativas estuvieron en desacuerdo con el desarrollo de la experiencia, y viceversa.

\section{Conclusiones e implicaciones.}

Una de las primeras conclusiones a las que llegamos con el desarrollo de este estudio es que los estudiantes indicaron una elevada satisfacción hacia las acciones formativas a través de la red, al valorar positivamente tanto las preguntas específicas como las preguntas del cuestionario que se le aplicó. Este hecho se hace más relevante considerando que el $37 \%$ de los alumnos estaban participando por primera vez en este tipo de acciones formativas. Estos resultados coinciden con los encontrados en otros trabajos tales como: Llorente (2008a), Llorente y Cabero, (2008), Barroso y Cabero (2010), Cabero (2010), Cabero y Llorente (2010), Alí y Ahmad (2011), Mirete, García y Sánchez (2011).

Tanto en los trabajos que acabamos de mencionar como en nuestro estudio, las plataformas utilizadas no fueron las mismas y sin embargo, en todos ellos se alcanza satisfacción por parte del alumnado al realizar este tipo de acciones formativas; es por ello que, coincidimos con Cabero y Llorente (2005), y podemos concluir que la plataforma es un medio mas no la razón para alcanzar el aprendizaje, y reflejándose como satisfacción.

Otro de los resultados de nuestro trabajo coincide con las aportaciones de otros autores (Cabero, 2010; Cabero \& Llorente, 2010; Means, B., Toyama, Y., Murphy, R., Bakia, M. \& Jones, K., 2010) en cuanto a que se logra una mayor interacción con sus profesores al cursar acciones formativas en las modalidades e-learning o blendedlearning que en la modalidad tradicional.

Los alumnos perciben como adecuados para la formación a través de Internet los siguientes elementos: que tienen a su disposición un fuerte volumen de materiales para su formación, y que

además pueden ser ofrecidos en distintos tipos de recursos como audiovisuales y multimedia; que facilita la interacción con sus profesores y compañeros a través de las herramientas de comunicación (chat, correo electrónico, foro,...) que ofrece la plataforma; que les permite flexibilidad temporal y espacial para trabajar en su asignatura, que esta modalidad les facilita su trabajo, y les ayuda a llevar la asignatura al día, entre otros. Muchos de estos elementos también fueron mencionados por otros autores, tales como 
Cabero (2010), Barroso y Cabero (2010) y Cabero y Llorente (2010).

Entre los elementos que el alumnado menciona como inadecuados en relación a su formación a través de la red se encuentran los siguientes: los tiempos de respuesta de las tutorías por parte del profesorado, contenidos demasiados extensos, poco tiempo para resolver las e-actividades, desconocimiento de algunas de las funcionalidades y poco uso de la plataforma por parte de los profesores, lentitud en la conexión, poca interacción con sus compañeros a través de la plataforma.

Otra de las conclusiones a las que podemos llegar es que la actitud con la que los estudiantes inician este tipo de acciones formativas condiciona los resultados alcanzados y las percepciones en relación a la dinámica llevada a cabo por los profesores/ tutores durante el desarrollo de las mismas.

Otra de las conclusiones a las que llegamos en nuestro estudio es que el papel del profesor/tutor es un factor clave de éxito de las acciones formativas realizadas a través de Internet y esto coincide con lo planteado por Cabero (2006), y además, concuerda con lo encontrado en otros trabajos (Cabero, 2010; Cabero \& Llorente, 2010). Es por ello que, para que este tipo de modalidades de formación sea una experiencia exitosa, es muy importante la implicación del profesorado, que ofrezca tutoría adecuada y oportuna, y que además proporcionen retroalimentación durante el desarrollo de las mismas, y esto es lo que ha resultado de la opinión del alumnado en este estudio. Por lo tanto, es necesario el que el profesorado además adopte medidas para motivar y ayudar a los estudiantes tanto al inicio como durante el desarrollo de la acción formativa.

\section{Referencias bibliográficas.}

Alí, A. \& Ahmad, I. (2011). Key Factors for Determinig Students'Satisfaction in Distance Learning Courses: A Study of Allama Iqbal Open University. Contemporary Educational Tecnology, 2(2), 118-134. Recuperado de http:/ /www.cedtech.net/past2.asp?numara $=22$

Barroso, J. \& Cabero, J. (Febrero, 2010). Valoraciones de los alumnos sobre el elearning en las Universidades Andaluzas. Edutec. Revista Electrónica de Tecnología Educativa, 31. Recuperado

de http://edutec.rediris.es/Revelec2/ $\mathrm{r}$ e $\mathrm{v}$ e 1 e c $31 /$ e d u t e c e31_valoraciones_alumnos_elearning_un iversidades_andaluzas.html

Cabero, J. (2006). Bases pedagógicas del e-learning. Revista de Universidad y Sociedad del Conocimiento (RUSC), 3(1), 1-9. Recuperado de http: //www.uoc.edu/rusc/ 3/1/dt/esp/cabero.pdf

Cabero, J. (Dir.) (2010). Usos del elearning en las Universidades Andaluzas: Estado de la situación y análisis de buenas prácticas. Sevilla: Grupo de investigación Didáctica de la Universidad de Sevilla.

Cabero, J. \& Llorente, M. C. (2005). Las plataformas virtuales en el ámbito de la teleformación. Revista Electrónica Alternativas de Educación y Comunicación. Recuperado de http:// www.ealternativas.edu.ar/

Cabero, J. \& Llorente, M. C. (2010). La experiencia formativa de los alumnos en el Campus Andaluz Virtual (CAV). Revista de Universidad y Sociedad del Conocimiento (RUSC), 7(2), 1-15.

Cabero, J., Llorente, M. \& Puentes, A. (2010). La satisfacción de los estudiantes en red en la formación semipresencial. 
Comunicar, 35, 149-157. doi: 10.3916/C352010-03-08

Hurtado de Barrera, J. (2010). Metodología de la Investigación. Guía para la comprensión holística de la ciencia. Bogotá: Sypal-Quirón.

Llorente, M.C. (2008a). Blended-learning para el aprendizaje en nuevas tecnologías aplicadas a la educación: un estudio de caso (Tesis doctoral inédita). Universidad de Sevilla. Sevilla.

Llorente, M.C. (2008b). Satisfacción del alumnado universitario en procesos de formación. En R. Roig, (Dir.). Investigación e innovación en el conocimiento educativo actual (pp. 259-279). Marfil: Alcoy.

Llorente, M.C. \& Cabero, J. (2008). La formación semipresencial a través de redes telemáticas (blended learning). Barcelona: DaVinci.

Means, B., Toyama, Y., Murphy, R., Bakia, M. \& Jones, K. (2010). Evaluation of evidencie-based practicesin online learning: A meta-analisys and review online learning studies. Washington: U.S. Department of Education.

Mirete, A., García, F. \& Sánchez, M. (Septiembre, 2011). Implicación del alumnado en la valoración de su satisfacción con las web didácticas. Edutec. Revista Electrónica de Tecnología Educativa , 37, 1-13. Recuperado de http://edutec.rediris.es/ $\mathrm{R}$ e v e 1 e c $2 / \mathrm{R}$ e v e 1 e c 37 / p d f / Edutece_n37_Mirete_Garcia_Sanchez.pdf

Muñoz Justicia, J. (2005). Análisis cualitativo de datos textuales con Atlas.ti.5. Barcelona: Universitat Autónoma de Barcelona.

Fecha de recepción: 30-05-2013

Fecha de evaluación: 14-06-2013

Fecha de aceptación: 25-06-2013

Píxel-Bit. Revista de Medios y Educación. $N^{o}$ 44. Enero 2014. ISSN: 1133-8482. E-ISSN: 2171-7966. doi: http://dx.doi.org/10.12795/pixelbit.2014.i44.15 